\title{
Suppression of Allograft Rejection with Soluble VEGF Receptor 2 Chimeric Protein in a Mouse Model of Corneal Transplantation
}

\author{
Takahiko Hayashi, ${ }^{1}$ Tomohiko Usui ${ }^{2}$ and Satoru Yamagami ${ }^{2}$ \\ ${ }^{1}$ Department of Ophthalmology, Yokohama Minami Kyosai Hospital, Yokohama, Japan \\ ${ }^{2}$ Department of Ophthalmology, University of Tokyo Graduate School of Medicine, Tokyo, Japan
}

\begin{abstract}
When a transparent cornea becomes opaque due to infectious diseases, trauma, or ophthalmic surgery, the impaired cornea is replaced with a donor cornea to improve visual function. In this corneal transplantation, the graft survival rate is comparatively high, partly because of lacking vascular and lymphatic vessel in cornea. However, the transplanted corneas sometimes become opaque if allograft rejection occurs. Suppression of allograft rejection is critical for favorable outcomes of corneal transplantation. The essential effects of endogenous monomeric soluble vascular endothelial growth factor receptors (VEGFRs) 1 and 2 have been reported in corneal angiogenesis and lymphangiogenesis. This study investigated the effects of dimeric soluble VEGFR2/Fc chimera protein on corneal allograft rejection for future clinical application. Allogeneic full-thickness corneal transplantation was performed in C57BL/6 to BALB/c mice. The recipients were treated by intrastromal injection of soluble VEGFR1/Fc chimera (sR1/Fc group), soluble VEGFR2/Fc chimera (sR2/Fc group), or human IgG1/Fc protein (IgG/Fc group) at 0 , 7 , and 14 days after surgery. Both hemangiogenesis and lymphangiogenesis were significantly suppressed in the corneas of the sR2/Fc group compared with the $\mathrm{IgG/Fc}$ group. All grafts failed due to corneal wound rupture in the sR1/Fc group. In the sR2/Fc group, respective donor-derived $\mathrm{MHC}$ class $\mathrm{II}^{+} / \mathrm{CD} 11 \mathrm{c}^{+}$cells and CD11b-positive macrophage infiltration were reduced in the DLNs and the corneas showing a negative delayed-type hypersensitivity, compared with the IgG/Fc group. Our findings demonstrate that soluble VEGFR2/Fc chimera protein efficiently suppresses corneal allo-rejection, while reducing hemangiogenesis and lymhangiogenesis, and immune-competent cell-trafficking and may be a powerful tool for corneal allograft survival.
\end{abstract}

Keywords: chimera protein; corneal transplantation; hemangiogenesis; lymphangiogenesis; vascular endothelial growth factor

Tohoku J. Exp. Med., 2016 May, 239 (1), 81-88. C 2016 Tohoku University Medical Press

\section{Introduction}

Full thickness corneal transplantation is the most successful of all solid tissue allotransplantation procedures in humans. When topical steroids are administered, the graft survival rate is almost $90 \%$ in low risk patients (Küchle et al. 2002). This high success rate may be partially explained by the lack of both lymphatics and blood vessels in the cornea (Ambati et al. 2006; Albuquerque et al. 2009). However, after high-risk corneal transplantation involving the presence of at least two quadrants of stromal vascularization and/or a history of graft rejection, the graft survival rate declines significantly to below $50 \%$, despite local and systemic immunosuppression (Maguire et al. 1994; Thompson et al. 2003). In the vascularized cornea, there is parallel growth of both blood vessels and lymphatics. In a mouse model of corneal transplantation, removal of the cervical draining lymph nodes (DLNs) has been shown to inhibit graft rejection, indicating that these nodes have an essential role in alloimmunity after corneal transplantation (Yamagami and Dana 2001; Yamagami et al. 2002). Migration of donor and host antigen-presenting cells to the host cervical DLNs accelerate sensitization to graft antigens (i.e., corneal alloimmunity may be dependent on a functional ocular-lymphatic axis) (Liu et al. 2002). Inhibition of corneal lymphangiogenesis, in addition to inhibition of angiogenesis, may therefore be the ideal therapeutic strategy for avoiding graft rejection.

Vascular endothelial growth factor (VEGF) plays an essential role in both angiogenesis and lymphangiogenesis.

Received November 28, 2015; revised and accepted May 9, 2016. Published online May 20, 2016; doi: 10.1620/tjem.239.81.

Correspondence: Takahiko Hayashi, M.D., Ph.D., Department of Ophthalmology, Yokohama Minami Kyosai Hospital, 1-21-1 Mutsuura

Higashi, Kanazawa-ku, Yokohama, Kanagawa 236-0037, Japan.

e-mail: htakahiko-aikou@umin.ac.jp

Satoru Yamagami, M.D., Ph.D., Department of Ophthalmology, University of Tokyo Graduate School of Medicine, 7-3-1 Hongo, Bun-

kyo-ku, Tokyo 113-8655, Japan.

e-mail: syamagami-tky@umin.ac.jp 
There are several members of the VEGF family. VEGF-A induces both angiogenesis and lymphangiogenesis by binding to the VEGF receptor 1 (VEGFR1) and VEGFR2, although the latter process is mainly driven via the binding of VEGF-C and VEGF-D to VEGFR2 and VEGFR3 (Carmeliet and Jain 2000). It has been reported that blocking the corneal lymphatic vessels that flow directly to the DLNs is effective in preventing allograft rejection (Chen et al. 2004; Cursiefen et al. 2004; Bachmann et al. 2008). Recently, two important findings in the cornea have reported that a soluble form of VEGFR1 (sVEGFR1) acts as a potent natural inhibitor of corneal angiogenesis, and a soluble form of VEGFR2 (sVEGFR2) prevents lymphangiogenesis and maintains an alymphatic cornea (Ambati et al. 2006; Albuquerque et al. 2009). In addition, the immunosuppressive effects of soluble VEGFR2 and VEGFR2/Fc chimera protein have been reported after corneal transplantation (Albuquerque et al. 2009).

We used chimeric proteins with consideration for future clinical application in this study, because chimeric proteins can be easily produced in large quantities and reduce the risk of a reaction to foreign antibodies from a non-human animal in therapeutic treatments. The present study was therefore performed to investigate the influence of VEGFR1/Fc and VEGFR2/Fc chimera proteins on corneal alloimmunity. The mechanisms involved and the outcomes of exogenous administration of VEGFR1/Fc or soluble VEGFR2/Fc chimera protein after corneal transplantation were assessed for possible clinical applications.

\section{Materials and Methods}

Animals

$\mathrm{BALB} / \mathrm{c}$ and $\mathrm{C} 57 \mathrm{BL} / 6$ male mice weighing 25-30 g (8-12 weeks old) were purchased from CLEA Japan (Tokyo, Japan). Each mouse was anesthetized by intramuscular injection of a mixture of ketamine $(4.0 \mathrm{mg})$ and xylazine $(4.0 \mathrm{mg})$ before the surgical procedures.

It was necessary to use animals to study the immunological techniques. Animals were handled in accordance with the Association for Research in Vision and Ophthalmology Resolution on the Ethical Use of Animals in Research (http://www.arvo.org/about_arvo/policies/statement_for_the_use_of_animals_in_ophthalmic_and_visual_ research/\#organizations). Every effort was made to minimize pain to the animals, and the protocol was approved by the local animal experimentation ethics committee.

\section{Full-thickness corneal transplantation}

Full-thickness corneal transplantation was performed using two fully mismatched [major histocompatibility complex (MHC) and minor antigen] strains of mice, i.e., C57BL/6 $\left(\mathrm{H}-2, \mathrm{I}_{-} \mathrm{A}^{\mathrm{b}}\right)$ mice as the donors and $\mathrm{BALB} / \mathrm{c}\left(\mathrm{H}-2, \mathrm{I}-\mathrm{A}^{\mathrm{d}}\right)$ mice as the recipients, as previously described (Niederkorn and Mellon 1996; Yamagami and Tsuru 1999; Yamada et al. 2005). All surgical procedures were performed under an operating microscope. Corneal grafts were observed by slit lamp biomicroscopy twice a week for 8 weeks after corneal transplantation. Eyes with complications such as cataract, loss of the anterior chamber, hyphema, or infection were excluded from the study. A total of
100 eyes underwent corneal transplantation, but five eyes were not used in this study due to the death of the animals from anesthetic overdose.

Soluble VEGFR2 (flk-1)/Fc chimera protein (R\&D Systems, Minneapolis, MN, USA), soluble VEGFR1 (flt-1)/Fc chimera protein (R\&D Systems), or isotype control human $\mathrm{IgG} 1 / \mathrm{Fc}$ protein (R\&D Systems) was injected into the corneal grafts with a 33-gauge needle at a dose of $5 \mu \mathrm{g}$ as described previously (Stechschulte et al. 2001). The grafted mice were divided into the following three groups: 1) a soluble VEGFR1/Fc chimera protein group (sR1/Fc group, $\mathrm{n}=10), 2$ ) a soluble VEGFR2/Fc chimera protein group (sR2/Fc group, $n=16$ ), and 3 ) an isotype control $\operatorname{IgG1} / \mathrm{Fc}$ protein group (IgG/Fc group, $\mathrm{n}=$ 19). Full-thickness excision of the cornea of a normal donor eye was performed using a 2.0-mm trephine (Inami, Tokyo, Japan), and the graft was transplanted to a recipient corneal bed (diameter, $1.5 \mathrm{~mm}$ ) with 8 or 10 interrupted 11-0 nylon sutures (Mani, Tochigi, Japan). Sutures were removed at 7 days after surgery. Postoperatively, the corneal grafts were assessed for opacity and neovascularization by using a scoring system that has been previously reported (Sonoda and Streilein 1992; Sano et al. 1997).

\section{Investigation of angiogenesis and lymphangiogenesis}

Mice were euthanized at 10 days after corneal transplantation. Corneal flat mounts were excised, rinsed three times in phosphatebuffered saline (PBS), and fixed in acetone for $1 \mathrm{~h}$. The corneas were then rinsed once in PBS, blocked with $3 \%$ bovine serum albumin (BSA)-PBS for 2 days, incubated overnight at $4{ }^{\circ} \mathrm{C}$ with rat antimouse CD31 antibody (1:333; Abcam, Cambridge, UK) and rabbit anti-mouse LYVE-1 antibody (1:333; Abcam), and stained with Alexa Fluor $^{\circledR}$ 594-conjugated anti-rat or Alexa Fluor ${ }^{\circledR}$ 488-conjugated antirabbit secondary antibodies. Finally, the double-stained corneal flat mounts were covered with mounting medium (Vector Laboratories, Burlingame, CA, USA) and examined under a fluorescent microscope (BH2-RFLT3 and BX50; Olympus, Tokyo, Japan). For each cornea, a whole mount image was assembled from 9 or 12 photomicrographs (depending on the corneal size) taken at $100 \times$ magnification. NIH ImageJ software was used for image analysis. Neovascularization was quantified by setting a threshold level of fluorescence, above which only vessels were depicted. Assessment of neovascularization was performed in a masked manner. The area covered with blood vessels or lymphatics was outlined, with the innermost vessel of the limbal arcade as the border. Before analyses, several filters were used to obtain modified grayscale images of the whole mount preparations. Blood vessels and lymphatics were detected by setting a threshold level that included the bright vessels and excluded the dark background. The mean vascularized area of the $\mathrm{IgG} / \mathrm{Fc}$-treated (positive control) whole mount corneas was defined as $100 \%$, and the vascularized area of each cornea was determined relative to this value (vessel ratio).

Immunohistochemical evaluation of the donor-derived $\mathrm{MHC}$ class $\mathrm{II}^{+}$/ $C D 11 c^{+}$cells in host DLNs and CD11 $b^{+}$cells in the grafted corneas

In the sR2/Fc group, corneal sections and DLNs were harvested at 3 and 10 days after grafting, fixed in acetone, frozen in an optimal cutting temperature compound (Sakura Fine Technical, Tokyo, Japan) in liquid nitrogen, rinsed three times in PBS, and fixed in acetone for $1 \mathrm{~h}$. For some experiments, $8-\mu \mathrm{m}$ sections of the DLNs were fixed in $4 \%$ paraformaldehyde instead of acetone. Fixation was done in $10 \%$ formalin when staining with hematoxylin and eosin (HE) for light 
microscopy. Sections were then rinsed once in PBS and blocked with $3 \%$ BSA-PBS, followed by staining with rat anti-mouse CD11b antibody (1:100; Abcam) and rabbit anti-mouse LYVE-1 antibody (1:100; Abcam), as well as with Alexa Fluor ${ }^{\circledR}$ 594-conjugated anti-rat or Alexa Fluor ${ }^{\circledR}$ 488-conjugated anti-rabbit secondary antibodies. Cervical DLNs were also stained with purified CD11c antibody (1:100; BD Biosciences Pharmingen, San Jose, CA, USA) and fluorescein isothiocyanate-conjugated mouse anti-I- $\mathrm{A}^{\mathrm{b}}$ antibody, and then were stained with tetramethylrhodamine isothiocyanate-conjugated goat anti-Armenian hamster IgG (1:100; Jackson ImmunoResearch Laboratories, West Grove, PA, USA). To avoid nonspecific staining, sections were blocked with anti-Tc receptor monoclonal (CD16/ CD32) antibody for $30 \mathrm{~min}$ before immunostaining for $2 \mathrm{~h}$ with the primary antibodies or isotype-matched control antibodies, with subsequent incubation with secondary antibodies for $1 \mathrm{~h}$. All staining procedures were performed at room temperature, and each step was followed by thorough washing in PBS three times for 5 min each. Finally, the sections were covered with mounting medium (Vector Laboratories) and analyzed using a fluorescent microscope (BH 2-RFLT 3 and BX 50; Olympus). At least five sections were examined for each tissue specimen from each animal. The mean \pm standard deviation (SD) $/ \mathrm{mm}^{2}$ was determined from five to ten fields of at least four DLN or corneal samples.

Donor-specific delayed type hypersensitivity (DTH) after corneal transplantation

Induction of a donor-specific DTH response after corneal transplantation was assessed by an ear swelling assay. Recipient BALB/c mice transplanted with $\mathrm{C} 57 \mathrm{BL} / 6$ corneas received an injection of $1 \times$ $10^{6}$ mitomycin C (Kyowa-Hakko Kogyo, Tokyo, Japan)-treated $\mathrm{C} 57 \mathrm{BL} / 6$ spleen cells into the left ear at 4 weeks after corneal grafting. Positive control mice received a subcutaneous injection of $1 \times$ $10^{7} \mathrm{C} 57 \mathrm{BL} / 6$ spleen cells at $1 \mathrm{wk}$ before injection of the pinna. Spleen cells treated with $0.1 \mathrm{mg} / \mathrm{mL}$ mitomycin C and PBS were used for immunization and as control cells, respectively. Injection of C57BL/6 spleen cells into the pinna was done to trigger DTH following immunization of mice with the same cells. Ear thickness was measured with an engineer's micrometer (Mitsutoyo, Tokyo, Japan) before and $24 \mathrm{~h}$ after the challenge. Then ear swelling was calculated as follows: $(24 \mathrm{~h}-0 \mathrm{~h}$ value for the test ear $)-(24 \mathrm{~h}-0 \mathrm{~h}$ value for the control ear) $\times 10^{-3} \mathrm{~mm}$. Each group contained a minimum of five mice.

\section{Statistical analyses}

The unpaired $t$-test was used to compare mean values as appropriate. All analyses were performed using the StatView statistical software package (Abacus Concepts, Berkeley, CA, USA). Graft survival rates were compared between groups using the log-rank test. Differences of the alloantigen-specific DTH response between two groups were determined using the Mann-Whitney U-test. The level of significance was set at $\mathrm{p}<0.05$ or $\mathrm{p}<0.01$.

\section{Results}

\section{Fate of the corneal grafts}

Up to postoperative $8 \mathrm{wks}, 14$ out of 16 corneas remained transparent in the sR2/Fc group treated with the soluble VEGFR2/Fc chimera protein (Table 1). Injection of soluble VEGFR2/Fc chimera protein led to significantly better graft survival at 8 wks compared with that in the IgG/ Fc-treated control group ( $88 \%$ vs. $47 \%, \mathrm{p}=0.02)$. In contrast to the opaque corneal grafts of the $\mathrm{IgG} / \mathrm{Fc}$ group with prominent neovascularization, grafts were transparent in the $\mathrm{sR} 2 / \mathrm{Fc}$ group. Immunohistochemical analyses showed neither edema nor infiltrating cells in the corneas of the sR2/Fc group at 4 wks after corneal transplantation, whereas numerous mononuclear cells were detected in both the corneal grafts and the anterior chambers of the $\mathrm{IgG} / \mathrm{Fc}$ group (data not shown). In the $\mathrm{sR} 1 / \mathrm{Fc}$ group treated with soluble VEGFR1/Fc chimera protein, neovascularization of the corneal grafts was not observed before suture removal, but all of the grafts subsequently failed due to wound rupture (Table 1).

\section{Donor-specific DTH}

Significantly higher DTH responses were detected in the positive control group and the $\mathrm{IgG} / \mathrm{Fc}$ group at $4 \mathrm{wks}$

Table 1. Survival and rejection rates at 8 weeks after corneal transplantation.

\begin{tabular}{ccc}
\hline & Graft survival (\%) & Graft failure $(\%)$ \\
\hline sR2/Fc group & $14 / 16(88)$ & $2 / 16(12)$ \\
IgG/Fc group & $9 / 19(47)$ & $10 / 19(53)$ \\
sR1/Fc group & $0 / 10(0)$ & $16 / 16(100)$ \\
\hline
\end{tabular}

The effects of intracorneal injection of soluble VEGFR2/Fc chimera protein on the survival of corneal allografts. BALB/c mice underwent orthotopic transplantation from $\mathrm{C} 57 \mathrm{BL} / 6$ donors in one low-risk eye. The recipients were treated with soluble VEGFR2/ Fc chimera protein, VEGFR1/Fc chimera protein, or IgG/Fc. Survival of the grafts in mice treated with soluble VEGFR2/Fc chimera protein was significantly greater than in the $\mathrm{IgG} / \mathrm{Fc}$-treated control group ( $88 \%$ vs. $47 \% ; \mathrm{p}=0.02)$. All grafts in the $\mathrm{sR} 1 / \mathrm{Fc}$ group failed due to corneal wound ruptures. 


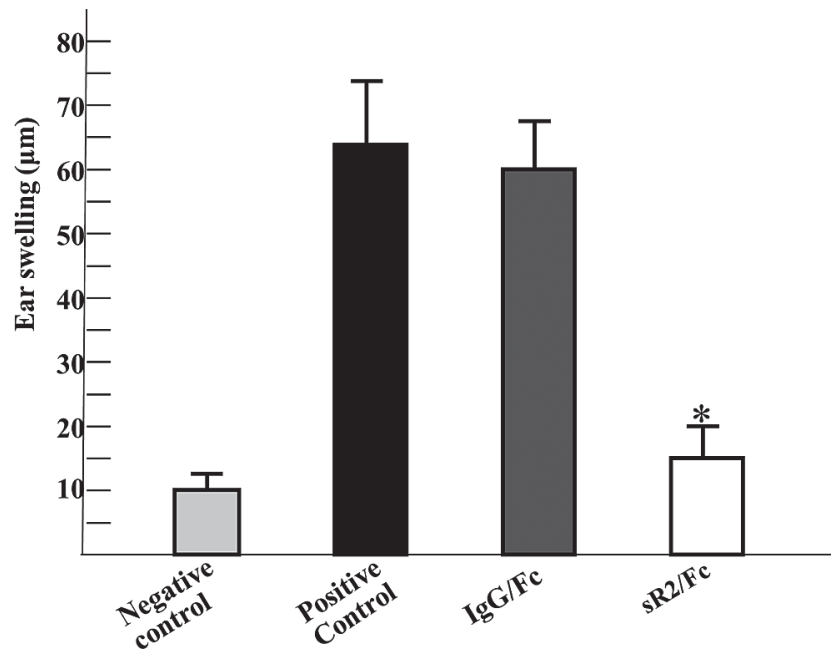

Fig. 1. Donor-specific delayed-type hypersensitivity (DTH).

The donor-specific DTH was tested in BALB/c mice at 4 wks after corneal transplantation. C57BL/6 spleen cells were injected into the pinna for the ear swelling assay. The DTH response of the $\mathrm{sR} 2 / \mathrm{Fc}$ group was negative. $(* \mathrm{p}<0.01$ vs. the positive control group).

A

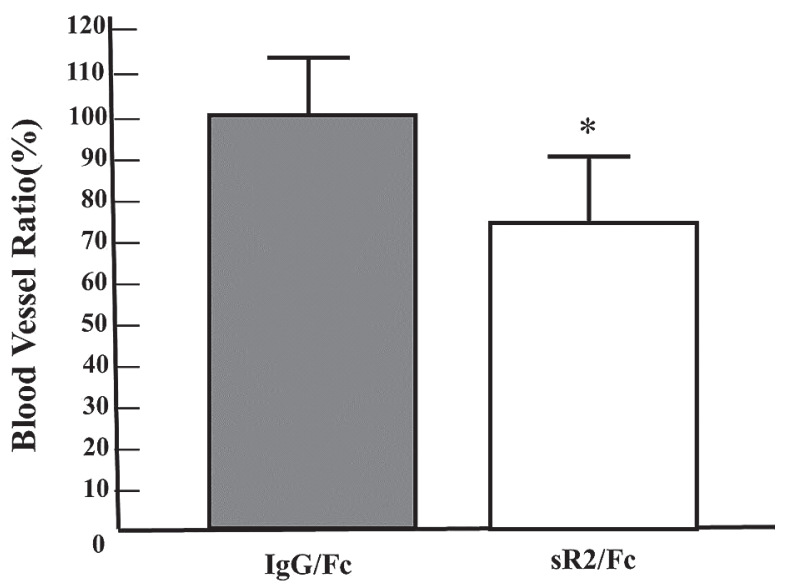

C

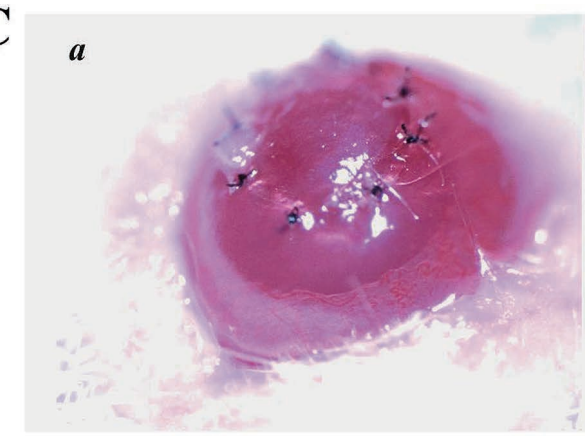

sR1/Fc, 7 days after corneal transplantation after corneal transplantation compared with the negative control group. In contrast, the DTH response in VEGFR2/ Fc group was negative (Fig. 1). Thus, administration of VEGFR2/Fc chimera protein led to inhibition of alloantigen-specific immune responses during the acute phase after corneal transplantation.

\section{Angiogenesis and lymphangiogenesis after corneal trans- plantation}

Because both angiogenesis and lymphangiogenesis are involved in corneal allograft rejection, we determined the effects of treatment with soluble VEGFR2/Fc chimera protein on these processes after PKP (Sonoda and Streilein 1992). A significant reduction of corneal graft neovascularization was found in the $\mathrm{sR} 2 / \mathrm{Fc}$ group, with angiogenesis and lymphangiogenesis being suppressed by $30 \%(\mathrm{p}<0.01)$ and $70 \%(\mathrm{p}<0.01)$, respectively, compared with the $\mathrm{IgG} /$ Fc group (Fig. 2A, B).

Fig. 2C shows corneas before suture removal in the sR1/Fc group and the $\mathrm{IgG} / \mathrm{Fc}$ group. The corneas were

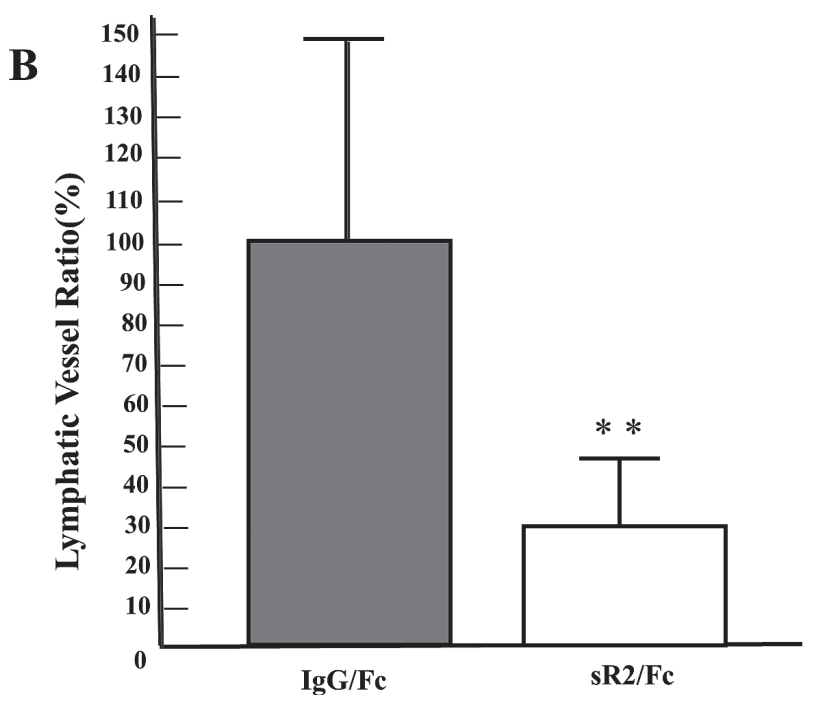

\section{$b$}

Fig. 2. Angiogenesis and lymphangiogenesis after penetrating keratoplasty.

(A) There were $30 \%$ fewer blood vessels in the $\mathrm{sR} 2 / \mathrm{Fc}$ group than in the $\mathrm{IgG} / \mathrm{Fc}$ group (both groups, $\mathrm{n}=16 ;{ }^{*} \mathrm{p}<0.01$ ).

(B) Lymphangiogenesis was inhibited by $70 \%$ in the sR2/Fc group (both groups, $\mathrm{n}=16 ; * * \mathrm{p}<0.01$ ). (C) The cornea had higher clarity in the sR1/Fc group (a) than in the IgG/Fc group (b). After administration of the soluble VEGFR1 chimera protein, wound healing was poor, and the graft was destroyed during the removal of the stitches. 
more transparent as compared to the $\mathrm{IgG} / \mathrm{Fc}$ group, without the presence of vascular invasion. In the sR1/Fc group, there was significant delay in wound healing, with the corneal structures destroyed by the suture removal. Unfortunately, observation was impossible because the tissue was disrupted and no tissue remained.

\section{Donor-derived MHC class II-expressing cells in the DLNS after corneal transplantation}

Cervical DLNs were harvested from BALB/c mice that received $\mathrm{C} 57 \mathrm{BL} / 6$ corneal grafts and were immunostained for detection of donor ( $\mathrm{I}-\mathrm{A}^{\mathrm{b}}$ ) derived $\mathrm{MHC}$ class II-expressing cells (green) and CD11c-expressing cells (red) (Fig. 3). Clusters of $\mathrm{I}-\mathrm{A}^{\mathrm{b}}$ cells were clearly visible in the DLNs of the IgG/Fc group, while such cells were decreased in the DLNs of the sR2/Fc group (Fig. 3A). The mean number of infiltrating MHC class II cells was significantly reduced by treatment with soluble VEGFR2/Fc chimera protein (Fig. 3B), presumably due to blockade of graftderived antigen-presenting cells in the DLNs $(p<0.01)$.

\section{Expression of CD11b and LYVE-1 in the donor corneas}

It was possible that the intrastromal injection of soluble VEGFR2/Fc chimera protein had an indirect effect on corneal angiogenesis and lymphangiogenesis after corneal transplantation, via suppression of CD11b-positive macrophages, which have been reported to be essential mediators of inflammatory corneal angiogenesis and lymphangiogenesis (Maruyama et al. 2005). The expressions of LYVE-1 (a marker of lymphatics) and CD11b in the donor corneas were therefore analyzed. In the $\mathrm{sR} 2 / \mathrm{Fc}$ group, there was significantly less recruitment of CD11b-positive cells, a significantly smaller area of lymphangiogenesis, and a significantly lower mean number of infiltrating CD11b-positive cells (Fig. 4A, C) ( $<<0.01)$. Similar findings of angiogenesis and lymphangiogenesis were obtained with flat mount preparations or by examination of corneal sections (data not shown). As shown by hematoxylin and eosin staining, in the sR2/Fc group, there was an absence of edema and infiltrating cells after transplantation. In the $\mathrm{IgG} / \mathrm{Fc}$ group, the corneal grafts showed numerous infiltrating cells, which caused opacity of the grafts (Fig. 4B).

\section{Discussion}

The present study shows that soluble VEGFR2/Fc chimera protein is a potent inhibitor of corneal angiogenesis and lymphangiogenesis, presumably by preventing the recruitment of CD11b-positive macrophages. Irreversible corneal graft failure may have multiple underlying mechanisms (Cursiefen et al. 2004). Antigen-presenting cells migrate to regional DLNs via lymph vessels after corneal transplantation, followed by induction of an immune response in the regional nodes (Yamagami and Dana 2001; Yamagami et al. 2002). Blocking the lymph flow to regional DLNs may therefore promote corneal graft survival, although this has not been reported previously in a clinical setting.

VEGFR2 and VEGFR3 are both closely associated with lymphangiogenesis (Carmeliet and Jain 2000). Intracorneal administration of soluble VEGFR2/Fc chimera protein could inhibit VEGF-C signaling via VEGFR3 expression on corneal dendritic cells, to prevent the migration of these cells to the DLNs. We hypothesize that intracorneal administration of the VEGFR2/Fc chimera protein may suppress corneal graft rejection after corneal transplantation via inhibition of lymphangiogenesis (Hamrah et al. 2003). In the present study, we showed that specific blocking of lymphangiogenesis with this protein suppressed allograft rejection after corneal transplantation in mice. CD11b-positive macrophages are involved in corneal lymphangiogenesis, promoted via the expression of various chemokines, cytokines, and growth factors (Maruyama et al. 2005; Shi et al. 2012). The present study showed that anti-lymphangiogenic therapy using soluble VEGFR2/Fc chimera protein inhibited both infiltration of the corneal grafts by CD11b-positive cells and trafficking of donorderived leukocytes to the DLNs.

We have shown that CD11b infiltration was lower in the sVEGFR2 group than in the Fc chimera control group (i.e., inflammation was suppressed). When inflammation occurs in the cornea, dendritic cells (CD11c positive) in the regional lymph nodes are activated. Soluble VEGFR2 suppresses VEGFR2 in the vascular endothelial cells or dendritic cells. As a result, the activation of CD11c in the RLN is suppressed. However, soluble VEGFR1 should strongly suppress macrophage infiltration by suppressing VEGFR1 in macrophages that are CD11b positive, because the macrophages express VEGFR1 and VEGFR3, but not VEGFR2. The inactivation of vascular endothelial cells or dendritic cells may reduce VRGFR1-related inflammation. Although the detailed mechanism of interaction is unknown, VEGFR2/Fc treatment reduces the inflammation of cornea and regional lymph nodes. Elucidation of this unknown mechanism should therefore be an aim of future studies.

In the present study, the administration of soluble VEGFR1/Fc chimera protein led to wound breakdown after corneal transplantation. Although anti-VEGF therapy is effective for blocking corneal neovascularization, delayed corneal wound healing is a significant problem (Dastjerdi et al. 2009; Kim et al. 2009). Although blocking of lymphangiogenesis alone may inhibit the induction of an immune response, blood vessels could still grow to supply the graft with nutrients and promote wound healing. Blocking of lymphangiogenesis, while allowing appropriate angiogenesis, may therefore be critical when establishing an effective therapeutic strategy for promotion of corneal allograft survival. Endogenous soluble VEGFR-2 is monomeric and thus only blocks VEGF-C and lymphangiogenesis, whereas the VEGFR2/Fc fusion protein is dimeric and can block VEGF-C as well as VEGF-A, to also inhibit angiogenesis. Exogenous administration of soluble VEGFR2 blocks lymphangiogenesis but not hemangiogenesis, as reported by 

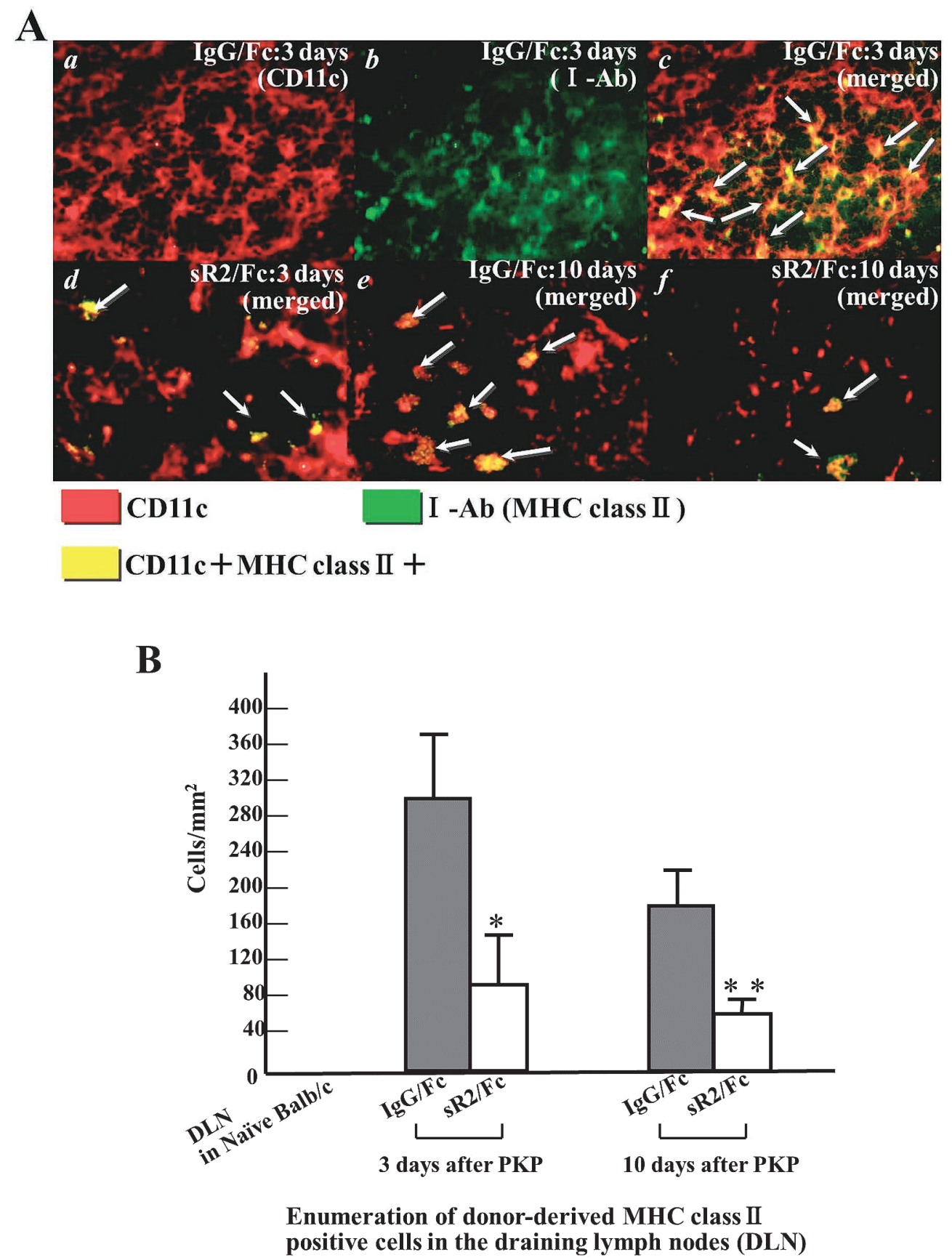

Fig. 3. Detection of donor-derived major histocompatibility class II antigen expression in the draining lymph nodes (DLNs) after penetrating keratoplasty (PKP).

(A) Immunohistochemical analysis of the DLNs confirmed the blockade of donor-derived antigen-presenting cells by treatment with sVEGFR2/Fc chimera protein. (a) CD11 $\mathrm{c}^{+}$cells (red) in a mouse from the IgG/Fc group at 3 days after PKP. (b) I-A $\mathrm{A}^{\mathrm{b}}$ positive cells (green) in the $\mathrm{IgG} / \mathrm{Fc}$ group at the same time as in (a). (c) Merged image of (a) and (b). (d) $\mathrm{CD} 11 \mathrm{c}^{+}$and $\mathrm{I}-\mathrm{A}^{\mathrm{b}+}$ cells in a mouse from the sR2/Fc group at 3 days after PKP. (e) IgG/Fc group at 10 days after PKP. (f) $\mathrm{sR} 2 / \mathrm{Fc}$ group at 10 days after PKP. Clusters of I-A ${ }^{\mathrm{b}}$ cells were clearly visible in the DLNs of the sR2/Fc group at 3 days (d) and 10 days (f) after PKP. Many more of these cells were detected in the IgG/Fc group (a-c). All I-A $\mathrm{A}^{\mathrm{b}}$ positive cells were colocalized with CD11c (a-c). Arrows indicate $\mathrm{MHC}$ class $\mathrm{II}^{+} / \mathrm{CD} 11 \mathrm{c}^{+}$double positive cells. (B) The mean number of infiltrating donor-derived MHC class II positive cells was significantly reduced by treatment with VEGFR2/ Fc chimera protein at 3 and 10 days after PKP $(\mathrm{p}<0.01)$.

other studies, whereas the present study shows that soluble VEGFR2/Fc chimera protein also has a significant inhibitory effect on angiogenesis, although the effect was not strong enough to adversely influence corneal wound healing
(Albuquerque et al. 2009). Thus, soluble VEGFR2/Fc chimera protein is a more "balanced" blocker of the growth of blood vessels and lymph vessels than either VEGFR1/Fc chimera protein or soluble VEGFR2 and may be more use- 

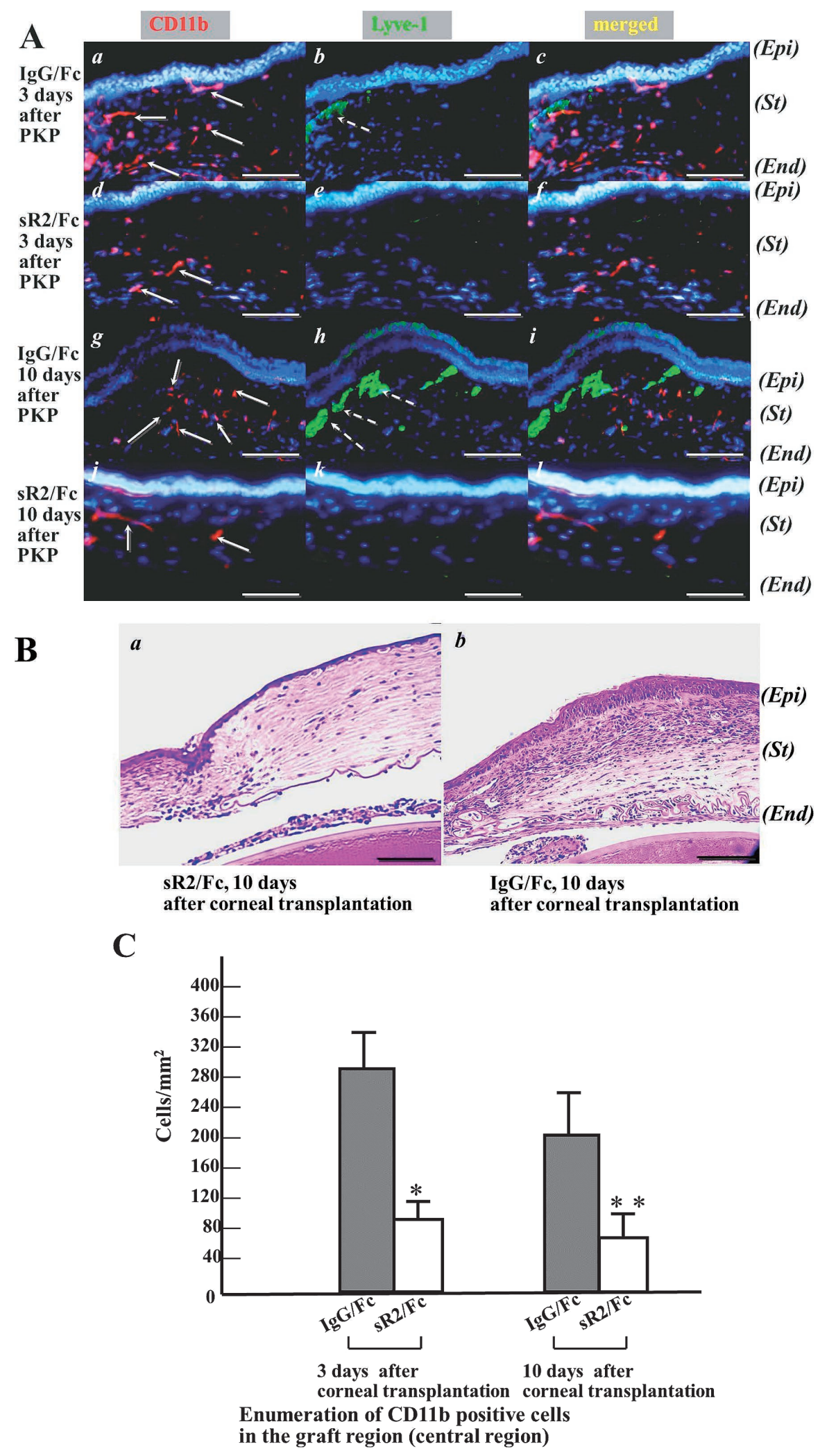

Fig. 4. Expression of CD11b and LYVE-1 in the donor cornea.

(A) After penetrating keratoplasty (PKP), mice from the sR2/Fc group had fewer CD11b-positive cells infiltrating the cornea compared with mice from the IgG/Fc group at both 3 days (a-f) and 10 days (g-l) after PKP. Results were similar to those obtained by staining of corneal flat mounts. LYVE- $1^{+}$lymphatics were not detected in the graft after treatment with the VEGFR2/Fc chimera protein (e, k). Nuclei were stained with 4',6-diamidino-2-phenylindole (blue). Epi, epithelium layer; St, stroma; End, endothelium. Arrows designate CD11b positive cells, and broken arrows designate LYVE-1 positive cells (bars $=100 \mu \mathrm{m}$; magnification, $\times 400$ ). (B) As shown by hematoxylin and eosin staining, there were fewer infiltration cells and less edema in the sR2 group than the $\mathrm{IgG} / \mathrm{Fc}$ group (bars $=100 \mu \mathrm{m}$; magnification, $\times 400$ ). (C) There was significantly less infiltration of macrophages into the donor corneas in the SR2/Fc group compared with the $\mathrm{IgG} / \mathrm{Fc}$ group $(\mathrm{p}<0.01)$. 
ful for preventing corneal graft rejection after corneal transplantation.

In summary, in addition to suppressing angiogenesis, local administration of soluble VEGFR2/Fc chimera protein prevents corneal graft rejection after corneal transplantation by suppressing lymphangiogenesis, macrophage infiltration, and donor-derived cell trafficking.

\section{Acknowledgments}

This work was supported by a grant from the Japanese Ministry of Health, Labor and Welfare, and a grant from The Japan Medical Association.

\section{Conflict of Interest}

The authors declare no conflict of interest.

\section{References}

Albuquerque, R.J., Hayashi, T., Cho, W.G., Kleinman, M.E., Dridi, S., Takeda, A., Baffi, J.Z., Yamada, K., Kaneko, H., Green, M.G., Chappell, J., Wilting, J., Weich, H.A., Yamagami, S., Amano, S., et al. (2009) Alternatively spliced vascular endothelial growth factor receptor-2 is an essential endogenous inhibitor of lymphatic vessel growth. Nat. Med., 15, 10231030.

Ambati, B.K., Nozaki, M., Singh, N., Takeda, A., Jani, P.D., Suthar, T., Albuquerque, R.J., Richter, E., Sakurai, E., Newcomb, M.T., Kleinman, M.E., Caldwell, R.B., Lin, Q., Ogura, Y., Orecchia, A., et al. (2006) Corneal avascularity is due to soluble VEGF receptor-1. Nature, 443, 993-997.

Bachmann, B.O., Bock, F., Wiegand, S.J., Maruyama, K., Dana, M.R., Kruse, F.E., Luetjen-Drecoll, E. \& Cursiefen, C. (2008) Promotion of graft survival by vascular endothelial growth factor A neutralization after high-risk corneal transplantation. Arch. Ophthalmol., 126, 71-77.

Carmeliet, P. \& Jain, R.K. (2000) Angiogenesis in cancer and other diseases. Nature, 407, 249-257.

Chen, L., Hamrah, P., Cursiefen, C., Zhang, Q., Pytowski, B., Streilein, J.W. \& Dana, M.R. (2004) Vascular endothelial growth factor receptor-3 mediates induction of corneal alloimmunity. Nat. Med., 10, 813-815.

Cursiefen, C., Cao, J., Chen, L., Liu, Y., Maruyama, K., Jackson, D., Kruse, F.E., Wiegand, S.J., Dana, M.R. \& Streilein, J.W. (2004) Inhibition of hemangiogenesis and lymphangiogenesis after normal-risk corneal transplantation by neutralizing VEGF promotes graft survival. Invest. Ophthalmol. Vis. Sci., 45, 2666-2673.

Dastjerdi, M.H., Al-Arfaj, K.M., Nallasamy, N., Hamrah, P., Jurkunas, U.V., Pineda, R. 2nd., Pavan-Langston, D. \& Dana, R. (2009) Topical bevacizumab in the treatment of corneal neovascularization: results of a prospective, open-label, noncomparative study. Arch. Ophthalmol., 127, 381-389.

Hamrah, P., Chen, L., Zhang, Q. \& Dana, M.R. (2003) Novel expression of vascular endothelial growth factor receptor (VEGFR)-3 and VEGF-C on corneal dendritic cells. Am. J. Pathol., 163, 57-68.
Kim, T.I., Chung, J.L., Hong, J.P., Min, K., Seo, K.Y. \& Kim, E.K. (2009) Bevacizumab application delays epithelial healing in rabbit cornea. Invest. Ophthalmol. Vis. Sci., 50, 4653-4659.

Küchle, M., Cursiefen, C., Nguyen, N.X., Langenbucher, A., Seitz, B., Wenkel, H., Martus, P. \& Naumann, G.O. (2002) Risk factors for corneal allograft rejection: intermediate results of a prospective normal-risk keratoplasty study. Graefes Arch. Clin. Exp. Ophthalmol., 240, 580-584.

Liu, Y., Hamrah, P., Zhang, Q., Taylor, A.W. \& Dana, M.R. (2002) Draining lymph nodes of corneal transplant hosts exhibit evidence for donor major histocompatibility complex (MHC) class II-positive dendritic cells derived from MHC class II-negative grafts. J. Exp. Med., 195, 259-268.

Maguire, M.G., Stark, W.J., Gottsch, J.D., Stulting, R.D., Sugar, A., Fink, N.E. \& Schwartz, A. (1994) Risk factors for corneal graft failure and rejection in the Collaborative Corneal Transplantation Studies. Ophthalmology, 101, 1536-1547.

Maruyama, K., Ii, M., Cursiefen, C., Jackson, D.G., Keino, H., Tomita, M., Van Rooijen, N., Takenaka, H., D’Amore, P.A., Stein-Streilein, J., Losordo, D.W. \& Streilein, J.W. (2005) Inflammation-induced lymphangiogenesis in the cornea arises from CD11b-positive macrophages. J. Clin. Invest., 115, 2363-2372.

Niederkorn, J.Y. \& Mellon, J. (1996) Anterior chamber-associated immune deviation promotes corneal allograft survival. Invest. Ophthalmol. Vis. Sci., 37, 2700-2707.

Sano, Y., Ksander, B.R. \& Streilein, J.W. (1997) Murine orthotopic corneal transplantation in high-risk eyes. Rejection is dictated primarily by weak rather than strong alloantigens. Invest. Ophthalmol. Vis. Sci., 38, 1130-1138.

Shi, V.Y., Bao, L. \& Chan, L.S. (2012) Inflammation-driven dermal lymphangiogenesis in atopic dermatitis is associated

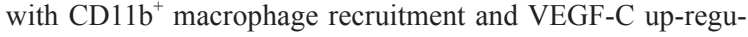
lation in the IL-4-transgenic mouse model. Microcirculation, 19, 567-579.

Sonoda, Y. \& Streilein, J.W. (1992) Orthotopic corneal transplantation in mice: evidence that the immunogenetic rules of rejection do not apply. Transplantation, 54, 694-704.

Stechschulte, S.U., Joussen, A.M., von Recum, H.A., Poulaki, V., Moromizato, Y., Yuan, J., D’Amato, R.J., Kuo, C. \& Adamis, A.P. (2001) Rapid ocular angiogenic control via naked DNA delivery to cornea. Invest. Ophthalmol. Vis. Sci., 42, 19751979.

Thompson, R.W. Jr., Price, M.O., Bowers, P.J. \& Price, F.W. Jr. (2003) Long-term graft survival after penetrating keratoplasty. Ophthalmology, 110, 1396-1402.

Yamada, J., Hamuro, J., Sano, Y., Maruyama, K. \& Kinoshita, S. (2005) Allogeneic corneal tolerance in rodents with long-term graft survival. Transplantation, 79, 1362-1369.

Yamagami, S. \& Dana, M.R. (2001) The critical role of lymph nodes in corneal alloimmunization and graft rejection. Invest. Ophthalmol. Vis. Sci., 42, 1293-1298.

Yamagami, S., Dana, M.R. \& Tsuru, T. (2002) Draining lymph nodes play an essential role in alloimmunity generated in response to high-risk corneal transplantation. Cornea, 21, 405-409.

Yamagami, S. \& Tsuru, T. (1999) Increase in orthotopic murine corneal transplantation rejection rate with anterior synechiae. Invest. Ophthalmol. Vis. Sci., 40, 2422-2426. 